weitgehend. Dennoch, der Leser nimmt als wertvolle Anregung mit, sich bei der Beurteilung von Rechtsregimen nicht nur mit den Vorzügen oder Nachteilen der einzelnen Regelungen zu befassen, sondern auch ihre gesellschaftlichen Rahmenbedingungen einzubeziehen.

Trotz der dargelegten Zweifel an einigen Wertungen wird das von Schiess Rütimann vorgelegte Werk zur Information über das belgische und besonders das Schweizer Parteienrecht mit seiner stupenden Informationsfülle wohl auf Jahre hin unverzichtbar sein.

Sebastian Roßner

\title{
Das Ende von New Labour: drei Ereignisgeschichten und ein Schuldiger
}

Mandelson, Peter: The Third Man, Harper Press, London 2011, 577 Seiten, £ 8,99.

Rawnsley, Andrew: The End of the Party. The Rise and Fall of New Labour, Penguin Books, London 2011, 895 Seiten, £ 12,99.

Seldon, Anthony und Guy Lodge: Brown at 10, Biteback Publishing, London 2011, 520 Seiten, $£ 14,99$.

New Labour - das war zunächst eine Erfolgsstory. Tony Blairs Wahlerfolg von 1997 wurde als Versprechen eines Neuanfangs, als der Beginn von Cool Britannia jenseits traditioneller Politik interpretiert. Was aus diesem Aufbruch wurde, ist heute noch umstritten. Patrick Diamond hat den widersprüchlichen Charakter von New Labour treffend zusammengefasst: „It was influenced by Thatcherism, explicitely rejected the traditional ethos of British social democracy, yet governed as previous Labour administrations had done, resdistributing the fruits of economic growth into public services and the welfare state. "1 Nicht vergessen werden darf, dass diese Politik, die sich selbst jenseits von Rechts und Links verortete, von Anfang an drei Konstruktionsfehler hatte: Sie machte die Labour Party zu einem Verbündeten der Globalisierung zugunsten der Finanzwelt, aber auf Kosten der Wählerschaft der Partei. Sie war unfähig mit den Herausforderungen durch langjährige Kriege (Irak, Afghanistan) umzugehen, und sie litt unter der Doppelregentschaft von Tony Blair und Gordon Brown. Anfangs wurde letztere durch eine Arbeitsteilung der beiden politischen Schwergewichte der Labour Party befriedet, und Gordon Brown konnte davon ausgehen, in absehbarer Zeit Nachfolger Tony Blairs zu werden. Seit 2003 wurde daraus eine offene Schlacht zweier Regierungslager um das Amt des Premierministers.

Was im Einzelnen geschah zeichnen zwei der hier zu betrachtenden Bände in höchster Detailgenauigkeit und auf der Basis von hunderten von Interviews nach. Anthony Seldon hat mit wechselnden Partnern zwischen 1990 und 2010 vier Bücher über die Amtsführung britischer Premierminister veröffentlicht. Er sieht sich der Zeitgeschichte verpflichtet und

1 Patrick Diamond, Governing as New Labour: An Inside Account of the Blair and Brown Years, in: Political Studies Review, 9. Jg. (2011), S. 145 - 162, S. 146. Vgl. auch Roland Sturm, Der „dritte Weg“" Demokratie im Zeitalter der Globalisierung, in: Uwe Backes / Eckhard Jesse (Hrsg.), Jahrbuch Extremismus \& Demokratie, 12. Jg., Baden-Baden 2000, S. 53 - 72. 
bietet deshalb eine chronologische Darstellung, deren Quintessenz in der Einleitung seines Bandes zur Amtszeit Gordon Browns zu finden ist. Andrew Rawnsley, erfahrener Journalist des Observer, verfährt ähnlich. Sein Zeithorizont ist indes weiter. Er beginnt die Nacherzählung der New Labour-Ära bereits in der Amtszeit Tony Blairs mit dessen zweitem Wahlsieg 2001. Peter Mandelsons Erinnerungsband ist nach Tony Blairs Darstellung seiner Amtszeit ${ }^{2}$ die zweite Aufarbeitung der New Labour-Ära, geschrieben von dem neben Blair und Brown „dritten Mann“ an der Spitze der Regierung. Mandelson machte eine wechselvolle Karriere durch. Zweimal, 1997 und 2001, musste er wegen Finanzskandalen von einem Ministeramt zurücktreten. 2004 nominierte ihn Blair als britischen EU-Kommissar. Und überraschenderweise holte sich Brown diesen Kopf des Blair-Lagers nach seiner Amtsübernahme als Premierminister 2008 zur Stärkung seines Kabinetts in die Regierung.

Endlich im Amt hätte Brown dieses nun gestalten können. Anthony Seldon und Guy Lodge skizzieren jedoch eine Persönlichkeit, die nicht mit sich im Reinen ist. Sie bescheinigen Brown exzellente Qualitäten in seiner ersten Zeit als Schatzkanzler von 1997 bis 2003. Danach habe er aber aufgehört, finanzpolitische Ideen zu entwickeln und konstruktiv zu arbeiten. Die Verpflichtung zur finanziellen Disziplin wurde aufgegeben, und Brown investierte seine Energie in den Machtkampf mit Blair. Die Autoren halten Brown als Premierminister für charakterlich nicht geeignet. Er war unfähig, im Team zu arbeiten und zu delegieren, weil er nur wenigen vertrauen wollte (insbesondere dem ökonomischen Sachverstand Ed Balls'). Seine Stärken bewies Gordon Brown in der Bankenkrise 2008. Die von ihm konzipierte Krisenstrategie fand weltweit Nachahmung. Im eigenen Land versuchte er aber zu lange, den Bürgern zu verschweigen, dass eine strikte Sparpolitik erforderlich sein würde. Bei der wahlentscheidenden Frage der Verbesserung öffentlicher Dienstleistungen (Gesundheit, Erziehung, Verkehr) führte er die New Labour-Agenda nur halbherzig fort. New Labour war für Brown weit weniger eine Vision einer anderen Politik als für Blair und seine Anhänger. Die New Labour-Rhetorik, die schon am Ende der zweiten Amtszeit Tony Blairs in den Hintergrund getreten war, verschwand nun in der Öffentlichkeit völlig. Brown machte sich mit seinen Wutausbrüchen nicht nur in der eigenen Regierung unbeliebt; er war auch, wie Umfragen dokumentierten, der unpopulärste Premierminister nach dem Zweiten Weltkrieg. Das Ende von New Labour wird von Seldon und Lodge also auf die thematische Auszehrung des New Labour Programms zurückgeführt. Die innenpolitischen Schwerpunkte dieses Programms wurden zudem durch die Kriegsallianz Tony Blairs mit George W. Bush überlagert, was den Unmut der Wähler weiter verstärkte. Großes Gewicht hatte aber auch das Versagen Gordon Browns als Premierminister und seine Zögerlichkeit, entweder mit vorgezogenen Neuwahlen oder einer Revitalisierung des New Labour-Programms einen Neuanfang zu wagen.

Für Andrew Rawnsley hat der Niedergang von New Labour vor allem damit zu tun, dass die Partei die fünf Erfolgsgaranten ihrer Politik nicht bewahren konnte. Erstens waren dies das Geschick und die Überzeugungskraft, mit der Tony Blair seine Politik vermitteln konnte. Er beherrschte den Umgang mit den Medien, und Rupert Murdochs Medienimperium unterstützte ihn. Heute wissen wir allerdings, was Rawnsley in diesem Zusammenhang nicht erwähnt: Diese Nähe hatte Züge einer illegalen Einflussnahme auf die Politik. Gordon Brown war nicht nur kein „Medienstar“. Er wurde auch im Wahlkampf 2010 mit der Situation 
konfrontiert, die Medien Murdochs wieder als Gegner zu haben. Der zweite Erfolgsgarant New Labours war der wirtschaftliche Aufschwung in Großbritannien und der damit verbundene feel-good-Faktor in der Wählerschaft. New Labour war in der glücklichen Lage, 1997 eine positive Wirtschaftsentwicklung von der Regierung John Majors zu „erben“. Der Aufschwung hielt bis zur weltweiten Bankenkrise an. Seither sind aber die alten Probleme des Landes, wie Defizite in der Bildungspolitik, vor allem in der beruflichen Bildung, Rückstände bei der Produktivität der Wirtschaft und die Abhängigkeit vom Wohl und Wehe der Londoner City, wieder überdeutlich geworden. Die dritte Stärke New Labours war die mangelnde Attraktivität der Opposition als politische Alternative. Die Konservativen galten seit den Zeiten Margaret Thatchers als die "nasty party“, die für die wahlentscheidende gut verdienende Mittelschicht nicht mehr wählbar war. David Cameron veränderte das Bild der Konservativen Partei in der politischen Auseinandersetzung mit New Labour zumindest zeitweise. Sein „mitfühlender“ Konservatismus ahmte das Blairsche Erfolgsrezept nach und machte es Brown schwerer, die eingefahrenen politischen Reflexe der Wählerschaft zu mobilisieren. New Labour hatte - viertens - anfangs erfolgreich die Lebensumstände vieler Bürger verbessert. Vom Frieden in Nordirland bis zum Mindestlohn, von der Verkürzung der Wartezeiten im National Health Service bis zum Ausbau von Schulen und der Neueinstellung von Lehrern reicht hier die (allerdings mit finanziell nicht ausreichend abgesicherter Ausgabenpolitik und Effizienzproblemen behaftete) Erfolgsbilanz. In der dritten Amtszeit New Labours stagnierte die Reform der öffentlichen Dienstleistungen, und der Unmut über deren Unzulänglichkeiten wuchs wieder. Die fünfte Säule des Erfolgsmodells war die Identifikation von New Labour mit Innovation, Erneuerung, Optimismus und Modernität. Sicherlich trugen hierzu auch die überragenden Marketingideen und -fähigkeiten der Medienspezialisten (spin doctors) und Think Tanks bei. Die Reduktion des Regierens auf Brot- und Butter-Themen durch Gordon Brown entsprach zwar dessen Politikverständnis und reflektierte seine Qualitäten, war aber wenig öffentlichkeitswirksam. Sein Versuch, durch Einbeziehen der „Politikverkäufer“ der Ära Tony Blair, Peter Mandelson und Alistair Campbell, dessen „Ideenfabrik“ neu zu nutzen, musste scheitern, weil seine inneren Überzeugungen zu wenig Raum für deren Strategien des Öffentlichkeitsmanagements ließen.

So waren Mandelsons Erinnerungen mit Spannung erwartet worden. Sie wurden zur großen Enttäuschung Browns. Peter Mandelson präsentiert sich darin, trotz seiner Nähe zu Brown in dessen Regierungszeit, als überzeugter New Labour-Mann. Nach seinem Bericht über die Revolte in der Spitze der Labour Party, die das Ziel hatte, Gordon Brown noch vor der Wahl zum Rücktritt zu bewegen, wusste jeder in der Labour Party, dass das größte Problem der Partei die Person des Premierministers war. Mandelson zitiert zustimmend Alistair Campbell: ,We all know what the real problem is. It's not because of what the government is doing. It's who is leading it." (S. 503)

Am Ende der drei Bände werden ausführlich die Details der Koalitionsverhandlungen der Labour Party mit den Liberaldemokraten 2010 geschildert. Gordon Brown wollte unbedingt die Chance einer "progressive coalition“ ergreifen, auch wenn sie eine wacklige Vielparteienzusammenarbeit im britischen Parlament erfordert hätte. Nur zögerlich sah er ein, dass er nicht Premierminister einer solchen Koalition würde bleiben können. Selbst als er sich zum Amtsverzicht durchgerungen hatte und Verhandlungen mit den Liberaldemokraten möglich wurden, blieb er der alte. Der wenig diplomatische Umgang mit den Liberaldemokraten, die inzwischen in die Rolle des Königmachers geschlüpft waren, führte in eine politische Sackgasse. In einem letzten Akt von Größe und Souveränität forderte Gordon Brown von Nick 
Clegg, dem Vorsitzenden der Liberal Democrats, fünf Tage nach der Wahl, sich zu entscheiden. Als dieser weiter lavieren wollte, reagierte Brown mit seinem sofortigen Rücktritt.

Das Ende von New Labour hatte mehr mit dem Scheitern eines politischen Projekts zu tun, das von seinen Kritikern als Thatcherismus mit menschlicherem Antlitz gesehen wurde - ein Projekt, das auch große Diskussionen über Manipulationen der Öffentlichkeit und die Macht der Medien provozierte und Fragen aufwarf nach dem Sinn der „special relationship“ mit den USA, die keineswegs mehr als Allianz auf Augenhöhe wahrgenommen wurde. Liest man die drei Bände, so sind sich die Autoren in einem einig: New Labour war zwar am Ende - aber dafür, dass der Fall dieser Idee so tief ausfiel und die Labour Party die Wahlen von 2010 verlor, gibt es einen Schuldigen: Gordon Brown. An seiner Intelligenz und Gradlinigkeit werden von keinem der Autoren die geringsten Zweifel geäußert. Er erscheint mehr als tragische Figur. In den Worten eines britischen Politikwissenschaftlers: aus dem finanzpolitischen „Stalin“ wurde in der Öffentlichkeit Mr Bean. ${ }^{3}$

Roland Sturm

3 Mit „From Stalin to Mr Bean“ ist ein Kapitel des Aufsatzes von Nicholas Allen überschrieben; ders., Labour's Third Term: A Tale of Two Prime Ministers, in: ders. / John Bartle (Hrsg.), Britain at the Polls 2010, London 2011, S. 1 - 36, S. 8.

\section{Rechtsextremismusforschung: einseitige Argumentation, verpasste Aufklärung}

Forum für kritische Rechtsextremismusforschung (Hrsg.): Ordnung. Macht. Extremismus. Effekte und Alternativen des Extremismus-Modells, VS Verlag für Sozialwissenschaften, Wiesbaden 2011, 376 Seiten, $€ 29,95$.

Weiß, Volker: Deutschlands Neue Rechte. Angriff der Eliten - Von Spengler bis Sarrazin, Verlag Ferdinand Schöningh, Paderborn 2011, 141 Seiten, € 16,90.

Extremismus taucht als Bedrohung der Demokratie und ihrer Werte in vielen Facetten auf. Gerade in Deutschland ist das Thema durch die „Schatten der Vergangenheit“ äußerst sensibel. Doch nicht nur in der Geschichte, sondern auch in der Gegenwart steht der demokratische Verfassungsstaat vor ständigen Herausforderungen, die seine Feinde heraufbeschwören. Momentan entfacht das Bekanntwerden eines Rechtsterrorismus eine um sich greifende Debatte über das offenkundige Versagen der Sicherheitsbehörden auch im Bundestag. Ein Untersuchungsausschuss soll die Vorgänge erhellen. Den Sozialwissenschaften kommt damit eine gesellschaftspolitische Herausforderung zu, die über die akademische Welt hinausgeht. Ein viel beachtetes Konzept ist dabei die normative Extremismustheorie, die die beiden deutschen Politikwissenschaftler Uwe Backes und Eckhard Jesse mit unzähligen Publikationen bis heute, unter anderem mit dem Jahrbuch Extremismus \& Demokratie, geprägt haben. ${ }^{1}$

1 Vgl. Uwe Backes / Eckhard Jesse, Demokratie und Extremismus. Anmerkungen zu einem antithetischen Begriffspaar, in: APuZ, B 44/1983, S. 3 - 18. 